\title{
La religion politique de Jean-Jacques : résolution d'un conflit entre universalisme et particularisme
}

Maxence Guillemin

\section{Q OpenEdition}

12 Journals

Édition électronique

URL : https://journals.openedition.org/rdr/638

DOI : $10.4000 /$ rdr.638

ISSN : 2534-7462

Éditeur

Presses universitaires de Strasbourg

\section{Édition imprimée}

Date de publication : 31 octobre 2017

Pagination : 107-128

ISBN : 978-2-86820-974-0

ISSN : 2493-8637

Référence électronique

Maxence Guillemin, «La religion politique de Jean-Jacques : résolution d'un conflit entre universalisme et particularisme », Revue du droit des religions [En ligne], 4 | 2017, mis en ligne le 15 janvier 2020, consulté le 23 mai 2022. URL : http://journals.openedition.org/rdr/638 ; DOI : https:// doi.org/10.4000/rdr.638

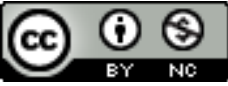

La revue du droit des religions est mise à disposition selon les termes de la Creative Commons Attribution - Pas d'Utilisation Commerciale 4.0 International - CC BY-NC 4.0. 


\section{LA RELIGION POLITIOUE DE JEAN-JACQUES: RÉSOLUTION D'UN CONFLIT ENTRE UNIVERSALISME ET PARTICULARISME}

\section{Maxence GUILLEMIN}

Docteur en droit public de l'Université de Reims Champagne-Ardenne

\section{RÉSUMÉ}

Cet article entend traiter du fait religieux intégré aux Institutions politiques de Jean-Jacques Rousseau. Celui-ci ne va pas de soi, pour qui se penche sur les rapports parfois ambigus que cultive le philosophe avec le spirituel. En imaginant l'irruption incidente d'une verticalité dans son Contrat social, Rousseau n'a pas manqué d'engendrer nombre de commentaires, qui traduisent une certaine perplexité, voire une simple condamnation. Celle-ci naît d'une apparente insolubilité : la nécessaire conciliation d'une religion totalement rationalisée, répondant au plus parfait universalisme, mais qui doit néanmoins être mise entre les mains du Législateur et donc s'inscrire dans une cité politique singulière. Ces développements reviennent sur ce déchirement permanent, véritable leitmotiv de la pensée du Genevois, et permettent de mieux saisir la notion de religion civile.

\section{ABSTRACt}

This article deals with religion, regarding Jean-Jacques Rousseau's Political Institutions. Religion is not self-evident, when the doctrine examines the ambiguous relationship between the philosopher and the spiritual. By imagining the irruption of verticality in his Social Contract, Rousseau generated many comments, which reflect a certain perplexity or even a simple condemnation. This condemnation is due to an apparent insolubility : the need to reconcile a religion totally streamlined, responding to the most perfect universalism, but which should nevertheless be put in the hands of the Legislator and therefore be part of a singular political city. These developments enlighten that permanent tear, as a leitmotif of the thought of Rousseau, and enable a better understanding of the concept of civil religion. 
T a simple évocation du fait religieux dans l'œuvre de Jean-Jacques Rousseau Ls'accompagne bien souvent d'une immédiate perplexité. Contrairement à une croyance largement répandue, le philosophe de Genève ne cantonne pas l'émergence d'un pacte social à une vision temporaliste, c'est-à-dire dépendante de la simple action des hommes. L'étonnement de voir la divinité jouer un rôle dans la formation du contrat est très certainement suscité par ce dogme de l'horizontalité que l'on rattache à Rousseau. Les hommes se rendant libres par l'attachement à un pacte commun, il aurait paru aller de soi qu'ils en fussent les seuls signataires. Pourtant, le contractualisme politique se heurte à une première difficulté : Rousseau connaît la propension des hommes à cultiver le fait religieux. Plus que cela, il sait que le cheminement de foi du religieux peut aisément coïncider avec la projection du sujet politique dans la citoyenneté. Rousseau est pour le moins explicite dans le Livre III de son Contrat social :

« Sitôt que les Hommes vivent en société, il leur faut une religion qui les y maintienne. Jamais peuple n'a subsisté ni subsistera sans religion, et, si on ne lui en donnait point, de lui-même il s'en ferait une ou serait bientôt détruit. »

Cette prise de conscience l'entraîne à ajouter à son ouvre du Contrat social un passage consacré à cette imbrication des sphères spirituelle et temporelle, à travers un chapitre intitulé « De la religion civile ${ }^{1}$ ». Là encore, la question d'une discontinuité avec l'ensemble de ses Institutions politiques se pose. Ceci est d'autant plus vrai que le passage sur la religion civile figure en toute fin du Contrat - remarque loin d'être anodine. En effet, Rousseau n'avait originellement pas joint ce chapitre lors du premier envoi de son œuvre à l'éditeur Marc-Michel Rey. La date de rédaction est incertaine, mais l'on sait que ce fut finalement quelques mois plus $\operatorname{tard}^{2}$, en hiver 1761, que Rousseau adressa à Rey cet ajout ${ }^{3}$. Ce qui apparaît certain, c'est que Rousseau considéra a posteriori que le concept de religion civile ${ }^{4}$ apportait des considérations inédites sur la prise en compte du facteur religieux

1. Rousseau J.-J., Du contrat social, Livre IV, Ch. VIII.

2. «Vous le trouvez petit pour un volume; cependant il est copié sur le brouillon que vous avez jugé devoir en faire un, et même le chapitre sur la religion y a été ajouté depuis. » : lettre à Marc-Michel Rey, 23 déc. 1761.

3. Waksman V., « Les difficultés étaient dans la nature des choses : de la religion de l'homme et du citoyen », in Waterlot G. (dir.), La théologie politique de Rousseau, Presse universitaire de Rennes, 2010, p. 92.

4. Rousseau semble établir comme synonymiques la «profession de foi civile» et la « religion civile », ce sont donc ces terminologies que nous adoptons indifféremment. 
par le Législateur, éléments que ce dernier ne pourrait ignorer ${ }^{5}$. Or, ces développements inédits s'inscrivent dans une dynamique bien particulière: Rousseau, au même instant de sa vie, traite des vertus de sa religion naturelle à travers la Profession de foi du vicaire savoyard, intégrée au Livre IV d'Émile ou De l'éducation. C'est bien à cette période que l'auteur revit le manuscrit du Contrat et en adopta la version définitive. Il s'agit là - on le comprend aisément - bien plus qu'une simple concomitance de temps. La publication jointe des deux textes n'est en rien anecdotique, si l'on veut admettre que Rousseau, "l'âme toute pénétrée » de la foi de son vicaire, a cru bon de revenir à sa théorie de religion civile pour lui apporter sa forme définitive ${ }^{6}$. Or, la double conceptualisation de la religion naturelle et de celle du citoyen présente, selon nombre d'auteurs, une véritable aporie. D'emblée, on se trouve face à une dichotomie classiquement rousseauiste, celle de l'état de nature et de l'ordre social. En se penchant sur le fait religieux, le lecteur peut se trouver face à deux chemins qu'il paraît mal aisé d'emprunter tout à la fois. L'un semble, en effet, exclure définitivement l'autre. Nous parlons ici de l'universalisme de la religion naturelle chez Rousseau, mis sous le prisme des particularismes de la cité politique. Pour autant, comprendre le rôle assigné par Rousseau à la religion doit nous éloigner d'une apparente impasse.

Le philosophe étudie dans son Contrat social les rapports traditionnels entre le politique et le religieux. Son constat est celui de l'échec, du rôle néfaste joué par le spirituel dans la cité. Par cela même, il provoque volontairement l'embarras du Législateur. Plus que jamais, la religion est un objet "nécessaire et impossible ${ }^{7}$ » dans la cité. Rousseau propose alors une profession de foi civile, dont la force conceptuelle serait de réunir le culte de l'homme et celui du citoyen. Il se propose d'en dessiner lui-même le cadre en en établissant les articles majeurs. Nous verrons que ceux-ci répondent de la simple raison universelle, tout en sanctifiant le contrat à l'échelle de la temporalité.

5. V. les notes de Simone Goyard-Fabre, in Rousseau J.-J., Du contrat social, Paris, H. Champion, 2010, p. 268.

6. Masson P.-M., La religion de Jean-Jacques Rousseau, Genève, Slatkine, 1970, facsimilé de l'éd. Hachette, 1916, p. 178.

7. GuÉNARD F., «"Esprit social" et "choses du ciel" : religion et politique dans la pensée de Rousseau », in WAterlot G. (dir.), La théologie politique de Rousseau, op. cit., p. 35. 


\section{LA RELIGION, OBJET NÉCESSAIRE ET IMPOSSIBLE}

Chacun sait que la pensée rousseauiste est dépendante d'une constante difficulté : comment libérer l'homme perverti par la société et l'ériger au rang de citoyen ? C'est en ce point précis que l'action humaine ne saurait être envisagée comme absolue.

Pour assurer la pérennité du contrat, Rousseau s'en remet aux mœurs, «partie inconnue à nos politiques [...] dont le grand Législateur s'occupe en secret ${ }^{8} »$. À ce titre, le Genevois développe longuement l'idée d'œuvre initiale du Législateur, dont il donne des indications quant à sa faculté de "persuader sans convaincre». L'auteur lui propose d'avoir recours à " une autorité d'un autre ordre » pour engendrer la communauté politique et donc concevoir le contrat. Dès lors, ce dernier n'est pas le fruit de la seule volonté générale, il est également la manifestation d'une intervention transcendantale. Le corps politique n'est donc pas simplement l'œuvre « du souverain, de la notion de loi, du gouvernement ${ }^{9}$ " tant que ce premier terme n'est pas doublé d'un recours à la verticalité ${ }^{10}$. En cela, le fonctionnement de la cité politique reste indissociable de la foi. L'émergence d'un théisme politique est une condition dont le Législateur ne peut faire fi, avec pour finalité d'asseoir l'autorité politique. Par ailleurs, Rousseau sait que la cité du Contrat ne peut se contenter du pacte créatif pour se pérenniser : il lui faut des lois. À ce titre, la notion d'intérêt général doit, une fois de plus, être vue comme relative. La cité politique - sans les lois aptes à traduire cette abstraction collective - n'est qu'un " corps sans âme ${ }^{11}$ " selon un passage du manuscrit de Genève. Or, les prodiges de la loi que Rousseau dépeignait déjà en 1755 lui avaient fait emprunter l'expression de « voix céleste » dans son Discours sur l'économie politique.

8. Rousseau J.-J., Du contrat social, Livre II, Ch. VII.

9. Waterlot G., La religion civile de Jean-Jacques Rousseau, conférence donnée aux Charmettes, 20 juin 2009, Première partie :

http://www.ac-grenoble.fr/PhiloSophie/old2/file/Conference_waterlot_charmettes_ juin_2009.pdf [consulté le 26 juin 2017].

10. « Pour qu'un peuple naissant pût goûter les saines maximes de la politique et suivre les règles fondamentales de la raison d'État, il faudrait que l'effet pût devenir la cause ; que l'esprit social, qui doit être l'ouvrage de l'institution, présidât à l'institution même; et que les hommes fussent avant les lois ce qu'ils doivent devenir par elles. Ainsi donc le législateur ne pouvant employer ni la force ni le raisonnement, c'est une nécessité qu'il recoure à une autorité d'un autre ordre, qui puisse entraîner sans violence et persuader sans convaincre. » : Rousseau J.-J., Du contrat social, Livre II, Ch. VII.

11. Rousseau J.-J., Du contrat social, $1^{\text {re }}$ version, Livre III. 
Ainsi, le rapport à la foi doit être mis à contribution pour servir la plus grande ambition de l'auteur, celle qui dirige la théorie première de ses Institutions politiques : en effet, il contribue à susciter la passion des lois et à élever l'homme en citoyen. Pour Rousseau, l'amour du devoir des hommes envers la communauté est la première condition à l'élévation du citoyen et de sa vertu ${ }^{12}$. Pourtant, le contrat originel n'est pas doté de force contraignante. À ce titre, Rousseau avait compris que la religion s'érigeait comme unique recours pour imposer les normes structurelles conduisant à l'éveil de l'esprit social, et in fine à la survie du contrat. De là découle la condition fondamentale pour que la liberté de l'homme lui soit rendue : les lois civiles ne peuvent à elles seules exacerber le sens du devoir ${ }^{13}$. La première cause est liée à leur simple caractère dissuasif, qui n'offre qu'une approche par la négative ${ }^{14}$. L'ordre dans la cité ne serait qu'illusoire, si le Prince n'attend de ses sujets qu'un simple rapport à l'obéissance. Le respect des lois n'est qu'un préalable nécessaire à l'amour de ceux qui s'y soumettent, ajouté au sens du devoir. Or, celui-ci échappe perpétuellement à la nature de l'homme, lequel verra dans le développement du «moi » une priorité naturelle. Il faudra dès lors transformer l'amour de soi en amour de l'ordre, et en cela, en amour de la vertu ${ }^{15}$. Si tel n'est pas le cas, le développement de l'individu s'exerce au détriment de la construction de la communauté politique $^{16}$. Cette propension inhérente aux individus peut alors être tempérée par d'autres passions. Toutefois, celles-ci ne peuvent répondre au simple cadre de la rationalité. Il faut alors générer une distorsion des jugements, tendant à prouver que l'intérêt général est une condition et un complément de l'intérêt particulier. Pour arriver à cette fin, le politique utilise des moyens "indirectement rationnels pour arriver à des fins rationnelles ${ }^{17}$ ». C'est que la religion suscite la passion, l'espérance et la crainte ${ }^{18}$. Le rapport à la transcendance façonne le développement de l'individu au-delà de tout

12. Celle-ci - contrairement à la bonté naturelle - ne peut que se déployer par l'acte communautaire; V. GUÉNARD F., précit., p. 26.

13. DÉChaux J.-H., "Rousseau et la médiation symbolique entre les hommes : contribution à un individualisme structurel », Sociologie, vol. 1, n 2, 2010, p. 278.

14. V. à ce sujet les développements de Ch. JACQUeT, La pensée religieuse de Jean-Jacques Rousseau, Louvain, Bibl. de l'université; Leiden, Brill, 1975, p. 162 et s.

15. WAKSMAN V., précit., p. 102.

16. TASSel C., « 3 clés pour comprendre Jean-Jacques Rousseau », Le Monde des religions, 16 févr. 2011.

17. Bachofen B., "La religion civile de Rousseau : une théologie politique négative », in Waterlot (dir.), La théologie politique de Rousseau, op. cit., p. 49.

18. La religion est présentée dans le manuscrit de Genève comme " une force intérieure qui pénètre jusqu'à l'âme et soit toujours indépendante des biens, des maux, de la vie et 
système politique et normatif ${ }^{19}$. Le respect et l'amour du devoir forgent le citoyen, la religion en est un outil de premier choix. En cela, l'utilisation de la foi demeure inhérente à l'action du Grand Législateur. Ainsi Rousseau cultive-t-il un dualisme des sources du droit, à la fois transcendantales et humaines, spirituelles et temporelles. En ayant recours à cette extériorité, à cette " autorité d'un autre ordre », Rousseau rompt définitivement avec le contractualisme des hommes : «le problème politique n'a pas ici de solution proprement politique ${ }^{20}$. »

L'intégration du fait religieux devient alors une arme pour le fondateur de la cité politique, qui doit exalter les particularismes en suscitant la passion des lois. Encore faut-il adopter la forme de culte appropriée. Pour cela, Rousseau se propose dans le Contrat de revisiter les rapports traditionnels entre le religieux et le politique. Le Genevois traite de deux extrêmes. D'une part, les formes d'ancien paganisme. Dans ce cas, la figure de souveraineté politique est entièrement liée au spirituel. D'autre part, Rousseau traite de la "religion de l'homme ", délestée de toutes les superstitions et inhérente à la conscience de chacun.

Dans son effort de catégorisation, Rousseau remonte tout d'abord au temps des sociétés primitives, dans lesquelles les royautés tiraient leur légitimité de la croyance en des personnages surnaturels et sacrés ${ }^{21}$. Pour l'auteur, « les hommes n'eurent point d'abord d'autres rois que de dieux, ni d'autre gouvernement que le théocratique ${ }^{22} »$. Ce constat le pousse à étudier les religions paiennes. Rousseau explique le lien qui existe entre un peuple et la religion qu'il s'est choisie, entraînant une interdépendance organique. Les hommes voyant en leur dieu leur roi, la théocratie était l'unique forme d'association politique. On retrouve là une idée phare de l'auteur : les hommes naissent libres, ils ne peuvent raisonnablement s'asservir qu'à travers la croyance d'obéir à un être surnaturel. Or les dieux n'étant - individuellement - reconnus que par la communauté qu'ils régissaient, il y eut tout

de tous les évènements humains » : Rousseau J.-J., Du contrat social, $1^{\text {re }}$ version, chapitre consacré au Législateur.

19. Bourdin C., «Autorité, pouvoir et service : la transcendance de la condition politique », Revue d'éthique et de théologie morale, $\mathrm{n}^{\circ} 244,2007 / 2$, p. 88.

20. GuÉNARD F., précit., p. 22.

21. Cette confusion totale entre sphères spirituelle et temporelle avait d'ailleurs été évoquée par Rousseau, précédemment dans le Contrat : "Si les hommes n'avaient pas cru au caractère surnaturel de leur chef, ils ne lui eussent point obéi. », in RouSSEAU J.-J., Du contrat social, Livre I, Ch. II.

22. Rousseau J.-J., Du contrat social, Livre IV, Ch. VIII, « De la religion civile». 
naturellement autant de dieux que de peuples. À ce titre, Rousseau n'hésite pas à reconnaître expressément de réels bienfaits sur la communauté politique. Ce culte fonde, incontestablement, les citoyens : « [La religion] est bonne en ce qu'elle réunit le culte divin et l'amour des lois, et que, faisant de la patrie l'objet de l'adoration des citoyens, elle leur apprend que servir l'État, c'est en servir le dieu tutélaire. »

De ce système, il en résulterait un dieu de la nation, catalyseur d'une projection très forte des individus dans la société. Toutefois, la première conséquence en serait l'« intolérance théologique et civile » selon Rousseau. En effet, deux peuples voisins ne pourraient ainsi être sous l'autorité d'un même chef. Rousseau n'ignore pas le caractère fanatique de tels citoyens. De cette intolérance, il en résulterait une violence sans égale ${ }^{23}:$ « [...] Alors mourir pour son pays, c'est aller au martyre ; violer les lois, c'est être impie ; et soumettre un coupable à l'exécration publique, c'est le dévouer au courroux des dieux : sacer esto.»

En tel domaine, l'exemple de la guerre est à-propos. Il démontre le caractère fanatisé des citoyens, dont Rousseau dresse le portrait dans sa critique de la religion paienne :

«[...] Une femme de Sparte avait cinq fils à l'armée, et attendait des nouvelles de la bataille. Un ilote arrive; elle lui en demande en tremblant : "Vos cinq fils ont été tués. - Vil esclave, t’ai-je demandé cela? - Nous avons gagné la victoire !" La mère court au temple, et rend grâces aux dieux. Voilà la citoyenne. ${ }^{24}$ "

C'est bien sur ce point précis que le Genevois introduit la diatribe. Il dénonce l'intolérance inhérente à une telle communauté politique vouée à la fanatisation. Un peuple «qui ne respire que meurtre et massacre, et croit faire une action sainte en tuant quiconque n'admet pas ses dieux ${ }^{25} »$ ne peut trouver grâce à ses yeux ${ }^{26}$. L'élévation de l'homme en citoyen ne peut se faire

23. Cette projection extrême de l'individu dans la communauté renvoie directement à des passages du premier livre d'Émile ou De l'éducation. Rousseau y donne les exemples d'attachement à la patrie qui dénaturent l'homme, lui ôtent son existence absolue pour lui en donner une relative, et transportent le moi dans l'unité commune. V. RoussEAU J.-J., Émile ou De l'éducation, Livre I, «L'âge de nature : le nourrisson (infans)».

24. Ibid.

25. Rousseau J.-J., Du contrat social.

26. Dans La profession de foi du vicaire savoyard, Rousseau traitait des dieux du paganisme comme des êtres «abominables [...] qui n'offraient pour tableau du bonheur suprême que des forfaits à commettre et des passions à contenter », in RousSEAU J.-J., Émile ou De l'éducation. 
à ce prix, sinon cultiver « un état naturel de guerre » contraire, ipso facto, à toute idée de pérennisation de la cité. Le second volet de cette critique a trait à l'instauration d'une morale que la religion doit cultiver chez Rousseau. L'accession au rang de citoyen dans une telle théocratie ne serait que la résultante d'une "sacralisation des convoitises humaines ${ }^{27}$ », et certainement pas un vecteur de vertu. Il reproche ainsi aux religions antiques d'être fondées sur "l'erreur et le mensonge ». Ce constat génère une défiguration de la notion de divinité et du culte qui lui est associé. Celui-ci s'en voit dès lors noyé dans un "vain cérémonial » qui entraîne la coupable superstition $^{28}$. Rousseau avait déjà développé cette dérive à travers un vieux texte rédigé très probablement dans la période 1753-1756 et publié bien après sa mort, en 1861. Il s'agit d'une allégorie dans laquelle l'auteur met en scène « un édifice immense formé par un dôme éblouissant que portaient sept statues colossales au lieu de colonnes ${ }^{29} »$. Celui-ci symbolise le paganisme. Ce temple abrite tous les peuples de la Terre:

« Directement au-dessous, c'est-à-dire au centre du bâtiment et au point de perspective, était un grand autel heptagone sur lequel les humains venaient en foule offrir leurs offrandes et leurs vœux aux sept statues qu'ils honoraient par mille différents rites et sous mille bizarres noms. Cet autel servait de base à une huitième statue à laquelle tout l'édifice était consacré et qui partageait les honneurs rendus à toutes les autres. Toujours environnée d'un voile impénétrable, elle était perpétuellement servie du peuple et n'en était jamais aperçue ; l'imagination de ses adorateurs la leur peignait d'après leurs caractères et leurs passions et chacun d'autant plus attaché à l'objet de son culte qu'il était plus imaginaire ne plaçait sous ce voile mystérieux que l'idole de son cœur. »

La clef de voûte de l'édifice majestueux révèle les termes : «Peuples servez les dieux de la Terre».

Les sept statues dont il est question symbolisent bien entendu les sept péchés capitaux. Le dieu qui surplombe le reste, jamais aperçu des humains qui lui vouent une divinisation sans égale, se voit appelé différemment en fonction des peuples. C'est bien le rôle du voile imaginé par Rousseau : le principe de l'amour intense de la patrie. Ce dieu des nations porte ainsi

27. Waterlot G., La religion civile..., précit., Deuxième partie, dont nous reprenons l'exemple du morceau allégorique sur la révélation et son enseignement.

28. V. JACQUet Ch., La pensée religieuse de Jean-Jacques Rousseau..., op. cit., p. 168.

29. Rousseau J.-J., Euvres et correspondance inédites, Paris, M. Lévy, 1861. Le texte a fait l'objet d'une publication plus récente, RousSEAU J.-J., « Fiction ou morceau allégorique sur la Révélation », in CEuvres complètes, t. II, Paris, Seuil, 1967-1971. 
dans sa main un cœur enflammé, et de l'autre un poignard. Provoquant la ferveur sans bornes des humains qui prient en son nom, cet amour est teinté de taches de sang. C'est que le dieu provoque l'hostilité envers les étrangers. Une fois le voile levé, la statue est reconnue comme "étouffant l'humanité personnifiée », la menant par la cruelle division qu'elle exerce à une perte certaine. Cette allégorie renvoie directement aux développements du Contrat social :

« [La religion païenne] est mauvaise en ce qu'étant fondée sur l'erreur et sur le mensonge, elle trompe les hommes, les rend crédules, superstitieux, et noie le vrai culte de la Divinité dans un vain cérémonial. Elle est mauvaise encore, quand, devenant exclusive et tyrannique, elle rend un peuple sanguinaire et intolérant, en sorte qu'il ne respire que meurtre et massacre, et croit faire une action sainte en tuant quiconque n'admet pas ses dieux. Cela met un tel peuple dans un état naturel de guerre avec tous les autres, très nuisible à sa propre sûreté ${ }^{30}$.»

La parabole proposée par l'auteur ne pouvait pas annoncer la fin de l'humanité sans possibilité de salut. Les faits allégoriques sont également une mise en perspective historique : ils tendent à raconter l'Histoire des hommes à travers la dérive de leurs croyances. Ce faisant, Rousseau introduit l'avènement de la chrétienté. Le temple païen, abritant l'humanité, sera le témoin de la venue de « ce personnage dont l'aspect imposant et doux frappa d'étonnement et de respect ${ }^{31} »$. Ses paroles, sous la plume du Genevois, annoncent le thème de recommencement du temps : "C'est ici le fils de l'homme. Les cieux se taisent devant lui, terre, écoutez sa voix. [...] O mes enfants, [...] je viens expier et guérir vos erreurs. Aimez celui qui vous aime et connaissez celui qui est. »

L'ère du paganisme n'est plus. L'affirmation d'un dieu de tous les hommes sera une rupture sans précédent dans l'Histoire de l'humanité : « [...] À l'instant saisissant la statue il la renversa sans effort et montant sur le piédestal avec aussi peu d'agitation, il semblait reprendre sa place plutôt qu'usurper celle d'autrui. »

Ainsi, pour Rousseau, l'avènement d'un dieu pour tous les hommes (son unicité et son universalité) répond d'une reconnaissance naturelle, qui ne tenait qu'au développement de la raison. En ce sens, le paganisme était antinomique avec toute idée de naturalisme.

30. Rousseau J.-J., Du contrat social.

31. Rousseau J.-J., « Fiction ou morceau allégorique sur la Révélation », art. cit. 
À travers cet avènement, Rousseau pourrait laisser entendre que la seule religion suscitant une vertu bénéfique à la communauté demeurerait celle de l'homme, sans artifices, sans perversions par le fait des princes ou des prêtres. Ce culte serait celui voulu par les Évangiles ${ }^{32}$, c'est-à-dire la formation d'une société vertueuse indépendante de la vision politique des souverains et libérée d'un ordre ecclésiastique qui pervertit la recherche de moralité ${ }^{33}$. Le phénomène doit très certainement interpeller d'autant plus l'observateur, que cette religion est, au fond, celle dépeinte par le Genevois dans la Profession de foi du vicaire savoyard, correspondant à l'intégralité du Livre IV d'Émile ou De l'éducation. Nous savons que l'œuvre en question fut publiée quelques semaines après le Contrat social. Cette concomitance présenterait une parfaite continuité de la pensée et des écrits de l'auteur. La religion du vicaire veut rétablir les «vérités de pratique ${ }^{34}$ » qui entraînent la recherche de vertu face aux dogmes spéculatifs, fruits des théologiens. Poursuivant sa critique, Rousseau réintroduit l'idée d'un théisme immaculé de toute action des hommes viciés :

« Reste donc la religion de l'homme ou le christianisme, non pas celui d'aujourd'hui, mais celui de l'Évangile, qui en est tout à fait différent. Par cette religion sainte, sublime, véritable, les hommes, enfants du même dieu, se reconnaissaient tous pour frères, et la société qui les unit ne se dissout pas même à la mort ${ }^{35}$."

C'est que les Évangiles se sont adressés - nous l'avons vu - à toutes les nations. Ce faisant, et pour Rousseau, ils ont voulu abolir toute idée de frontières. Leur message est ainsi purement opposé à l'ère du paganisme. Cela dit, le phénomène est exacerbé ${ }^{36}$, « la charité chrétienne ne permet pas de faire une différence odieuse entre le compatriote et l'étranger ${ }^{37}$ »; « le vrai chrétien ne regarde point l'étranger comme étranger mais comme son frère, il ne regarde point l'ennemi comme ennemi mais comme son prochain $^{38} »$.

32. «[Religion] sans temples, sans autels, sans rites, bornée au culte purement intérieur du Dieu suprême et aux devoirs éternels de la morale, est la pure et simple religion de l'Évangile, le vrai théisme, et ce qu'on peut appeler le droit divin naturel. » : RousSEAU J.-J., Du contrat social.

33. Dans ce chapitre du Contrat, Rousseau se propose également de faire la critique du christianisme historique, religion de Rome.

34. Rousseau J.-J., Émile ou De l'éducation, Livre IV : La profession de foi du vicaire savoyard.

35. Rousseau J.-J., Du contrat social.

36. Waterlot G., Rousseau : religion et politique, Paris, PUF, 2004, p. 65 et s.

37. Rousseau J.-J., Lettre à Usteri, 30 avril 1763.

38. Rousseau J.-J., Lettres écrites de la montagne, feuillet 70. 
Or ce constat pose problème aux yeux de Rousseau, lorsque la religion de l'homme se voit transposée dans le champ politique. On voit aisément apparaître la difficulté. La communauté politique voulue par le théoricien induit la nécessité d'un contrat exclusif. L'universalité et le moralisme attachés à l'acte religieux ne peuvent détruire ce dernier, sous peine de trahir le pacte. La pérennité de la cité passe par une prise en considération sans réserve de l'étranger, préalable indispensable pour pouvoir identifier les membres du contrat. La multiplication des échanges entre citoyens est bonne pour la conservation de la communauté, elle doit chez Rousseau demeurer le fruit du volontarisme politique du Prince. En revanche, l'interdépendance avec l'étranger au-delà du simple fait politique est néfaste pour la cité. Le lien social s'en voit fatalement amoindri et affaiblit la construction abstraite que Rousseau se fait du citoyen. Or ce dernier - dans la rhétorique du Genevois - doit être une finalité permanente dans l'action de l'homme, un critère fondamental du développement du soi. Dans une vision très déterministe, l'éveil de l'individu puis son élévation doivent être en tout état de cause conditionnés par les particularismes attachés à la communauté ${ }^{39}$. Le sens de l'intérêt général est exclusiviste, il ne peut s'accommoder du seul universalisme. Celui-ci est exacerbé par «la religion de l'homme». Ce faisant, il affecte tout au même moment l'action souveraine des membres $\mathrm{du}$ contrat. Rousseau est très explicite dans la première Lettre écrite de la montagne, rédigée deux années plus tard : « [...] Bien loin de taxer le pur Évangile d'être pernicieux à la société, je le trouve, en quelque sorte, trop sociable, embrassant trop tout le genre humain pour une législation qui doit être exclusive; inspirant l'humanité plutôt que le patriotisme, et tendant à former des hommes plutôt que des citoyens ${ }^{40}$."

Cette inaptitude naturelle à caractériser l'ennemi rend les chrétiens impuissants à protéger la patrie. Rousseau projette le constat dans le champ belliqueux ${ }^{41}$. Selon lui, l'attente du salut et la remise en une providence demeurent incompatibles avec la formation du bon soldat. Les chrétiens «marchent sans peine au combat; nul d'entre eux ne songe à fuir ; ils font leur devoir, mais sans passion pour la victoire; ils savent plutôt mourir que vaincre ». Face à des armées « que dévore l'ardent amour de la gloire et de la patrie », les défenseurs de la nation seraient « battus, écrasés, détruits, avant

39. « On nous dit qu'un peuple de vrais chrétiens formerait la plus parfaite société que l'on puisse imaginer. Je ne vois à cette supposition qu'une grande difficulté : c'est qu'une société de vrais chrétiens ne serait plus une société d'hommes. » : Rousseau J.-J., Du contrat social.

40. Rousseau J.-J., Lettres écrites de la montagne, première lettre, 1764.

41. V. TAssel C., art. précit. 
d'avoir eu le temps de se reconnaître ». De plus, la menace peut aussi venir de l'intérieur, ce qui pervertirait tout autant le système politique. C'est que, pour Rousseau, les termes de "république chrétienne » forment l'oxymore de l'impossible liaison. Pour que le régime se déploie, il faudrait que la vertu dépasse la simple religion des hommes pour envahir le champ politique. Or, pour le Genevois, la notion de «bon chrétien » ne peut être pensée en globalité, il y aura toujours un "Catilina, un Cromwell ${ }^{42}$ », celui-là même qui saura profiter du pieux aveuglement de ses compatriotes. Le déploiement de l'homme dans la sphère spirituelle provoque l'inaction dans la sphère temporelle, doublée d'un amour immodéré et asservissant de son prochain. Ainsi, le Prince usurpateur, " dès qu'il aura trouvé par quelque ruse l'art de leur en imposer et de s'emparer d'une partie de l'autorité publique, voilà un homme constitué en dignité ». Rousseau réintroduit l'idée de dualisme, d'une symbiose impossible entre la moralité du chrétien et la recherche du bien commun républicain, c'est-à-dire de la volonté générale. Sur ce point, il rejoint le constat de Machiavel $^{43}$ : « le vrai christianisme ${ }^{44}$ », « institution de paix », ne peut former de bons citoyens.

Ainsi la religion naturelle et le vieux paganisme - inscrits dans la cité politique - sont traités par Rousseau dans le Contrat social, et font l'objet d'une critique radicale. L'un dans l'autre, le philosophe ne peut que constater l'impuissance du souverain à user de ces formes de culte pour pérenniser la cité politique. En mettant en directe comparaison les dérives de la religion de l'homme et de celle du citoyen, Rousseau poursuit sa tentative d'établir la forme de culte idéale. Celle-ci, objet "nécessaire et impossible » dans la cité, est plus que jamais prise dans l'étau abyssal de l'universel et du particulier.

La formule est lapidaire : "considérant la religion par rapport à la société ", Rousseau estime que « toutes, à bien prendre, sont nuisibles à l'État ${ }^{45}$ ». Après avoir présenté les incompatibilités manifestes du spirituel et du temporel, il prend à partie le lecteur pour une bien singulière constatation : aucune religion ne convient à sa cité politique. Toutefois, sa présence demeure pour Rousseau un critère constitutif fondamental. L'auteur aborde alors une césure

42. Rousseau J.-J., Du contrat social, Livre IV, Ch. VIII.

43. L'influence de ce dernier sur Rousseau demeure importante. Le Florentin avait développé cette idée dans le Discours sur la première décade de Tite-Live, notamment dans le Livre II, Ch. II., puis le Livre III, Ch. I.

44. Rousseau J.-J., Lettres écrites de la montagne, première lettre, 1764.

45. Rousseau J.-J., Du contrat social, Livre IV, Ch. VIII. 
radicale $^{46}:$ « Laissant à part les considérations politiques, revenons au droit, et fixons les principes sur ce point important. »

Rousseau renvoie au Législateur la prérogative de caractériser la religion officielle au sein de la cité. Seul le corps politique pourra ainsi en fixer les $\operatorname{articles}^{47}$. Toutefois, ce même Législateur semble dès lors se trouver dans une inévitable impasse. Le Genevois pallie cet écueil en ajoutant à sa religion naturelle un dogme unique, qui en modifiera totalement la substance et suscitera une création conceptuelle.

\section{LA RELIGION COMME CEUVRE DU LÉGISLATEUR}

Rousseau - on le sait - part du préalable que l'amour de l'ordre ne peut s'exonérer de la foi. Par ailleurs, il connaît la propension naturelle des hommes à se référer au fait religieux. Ce double constat entraîne le philosophe à user de formules quelque peu lapidaires. Après la césure que nous venons d'évoquer, Rousseau impose la force de la vérité révélée : «Il y a donc une profession de foi purement civile dont il appartient au souverain de fixer les articles, non pas précisément comme dogmes de religion, mais comme sentiments de sociabilité sans lesquels il est impossible d'être bon citoyen ni sujet fidèle ${ }^{48}$. "

La profession de foi est d'emblée définie par le Genevois comme «purement civile », manière de démontrer que l'État ne se départit pas de ses facultés légitimes. Or ce procédé légitimant est capital pour Rousseau, il s'en expliquera dans la Première lettre écrite de la Montagne en 1764. La raison seule doit juger du "vrai, faux ou douteux ${ }^{49}$ ", matière sur laquelle «les tribunaux humains ne peuvent étendre leur compétence ${ }^{50} »$. En revanche, la «partie de la religion qui regarde la morale, c'est-à-dire la justice, le bien public, l'obéissance aux lois naturelles et positives, les vertus sociales, et tous les devoirs de l'homme et du citoyen ${ }^{51} »$ relève du gouvernement, elle « rentre directement sous sa juridiction ${ }^{52} »$. Aussi, l'emploi de cette pro-

46. Waterlot G., La religion civile..., précit., Deuxième partie.

47. Desmons É., « Réflexions sur la politique et la religion, de Rousseau à Robespierre », Rev. française d'histoire des idées politiques, $\mathrm{n}^{\circ} 29,2009 / 1$, p. 79.

48. Rousseau J.-J., Du contrat social, Livre IV, Ch. VIII.

49. Rousseau J.-J., Lettres écrites de la montagne, première lettre, 1764.

50. Ibid.

51. Ibid.

52. Ibid. 
fession de foi s'accompagne de bornes bien définies : celle-ci doit tendre à l'éternelle recherche du bien commun. Les articles de la religion civile ne s'érigent donc pas en dogmes religieux stricto sensu ${ }^{53}$, mais en "maximes sociales $^{54}$ » ou en « sentiments de sociabilité $e^{55} »$.

Même s'il revient au Législateur de déterminer la teneur exacte de ces articles, le Genevois donne des indications ${ }^{56}$. Les articles, selon Rousseau, doivent échapper aux dogmatismes inhérents aux religions positives, d'où leur petit nombre et leur accomplissement tempéré par la raison seule. On retrouve ainsi dans le Livre IV, Chapitre VIII du Contrat une énumération des articles que Rousseau propose au Législateur. Ceux-ci sont brefs : l'existence d'une "Divinité puissante, intelligente, bienfaisante, prévoyante et pourvoyante, la vie à venir, le bonheur des justes, le châtiment des méchants ${ }^{57}$ ». Or, d'emblée, les termes choisis frappent l'observateur. En effet, ces dogmes sont communs avec ceux du Vicaire savoyard, ils répondent à la religion naturelle décrite par Rousseau. L'existence d'un théisme vecteur de vertu est la pierre angulaire de la religion du Vicaire. La croyance en une survivance de l'existence était elle aussi mentionnée dans la Profession de foi du vicaire savoyard. Là encore, seul l'accomplissement des dogmes de la raison ${ }^{58}$ est présenté par Rousseau comme le préalable auquel chaque homme doit s'adonner. La «vie à venir » se refuse à celui qui « use mal de la raison qu'il a cultivée » et «il n'y aura d'incrédules punis que ceux dont le cœur se ferme à la vérité ${ }^{59} »$. De la même manière, l'individu non perverti par l'action des

53. JACQUet Ch., La pensée religieuse de Jean-Jacques Rousseau..., op. cit., p. 175.

54. On retrouve là l'expression de Rousseau, écrivant à Voltaire dès 1756. Le terme provient de la lettre sur la Providence : "Je voudrois donc qu'on eût dans chaque État un code moral, ou une espece de profession de foi civile qui contînt positivement les maximes sociales que chacun seroit tenu d'admettre, \& négativement les maximes intolérantes qu'on seroit tenu de rejetter, non comme impies, mais comme séditieuses » : RoussEAU J.-J, Lettre à Voltaire «sur la Providence », 18 août 1756.

55. Rousseau J.-J., Du contrat social, Livre IV, Ch. VIII.

56. Le caractère novateur de cette profession de foi n'est jamais explicité par Rousseau. Selon nombre d'auteurs, le fait de prétendre engendrer une religion sui generis était tout bonnement inenvisageable, vu le contexte de publication, voire dangereux pour l'auteur. Rousseau rappelle dans sa première lettre écrite de la montagne que le Conseil aux représentations des citoyens et bourgeois de Genève a, en 1762, considéré ses livres comme «impies, scandaleux, téméraires, pleins de blasphêmes \& de calomnies contre la Religion. Sous l'apparence des doutes, l'Auteur y a rassemblé tout ce qui peut tendre à sapper, ébranler \& détruire les principaux fondemens de la Religion Chrétienne révélée ».

57. Rousseau J.-J., Du contrat social, Livre IV, Ch. VIII.

58. Sur la révélation par la raison des sentiments moraux, V. DÉCHAux J.-H., art. cit., p. 276.

59. Rousseau J.-J., Émile ou De l'éducation, Livre IV. 
hommes ne pourra qu'être sauvé : « Nous tenons que nul enfant mort avant l'âge de raison ne sera privé du bonheur éternel. » La projection de cette espérance de salut peut être assez aisément transposée dans le champ politique. Comme souvent, il est question de rhétorique. Si l'individu ne croit pas à l'existence d'une vie à venir, il ne peut autoriser sa raison à accepter les sacrifices voulus par la communauté ${ }^{60}$. Cette condition demeure également une constante de l'auteur. Il en développe longuement l'idée dans le Contrat. L'élévation de l'individu en citoyen implique une projection de soi pleine et entière, laquelle peut parfois induire le don de sa vie pour la cité61 Or, l'absence de toute vision d'une vie future rend d'autant plus fragiles cet attachement et cette loyauté démesurés. On sait que c'est ce point précis qui força - selon le Genevois - les hommes à user de la religion dans la sphère politique. Le premier des sacrifices demeure bien entendu le don de sa vie en temps de guerre. Pour autant, nous savons que l'espérance d'une vie éternelle avait déjà été traitée par Rousseau dans sa critique de la religion de l'Évangile comme religion politique. L'attente du salut s'imposait alors en obstacle à l'élévation de l'homme en citoyen et à la projection de son Moi dans la cité. Rousseau semble surmonter cette difficulté à travers un dernier dogme positif de sa profession de foi civile : celui de la «sainteté du contrat social et des lois ${ }^{62} »$. Il s'agit là du seul dogme ne figurant pas dans Émile. Les termes de "sainteté du contrat » étaient déjà présents, en revanche, dans le Contrat, en son Chapitre III portant sur la fondation de la cité politique et la portée du pacte originel : «[...] Mais le corps politique ou le souverain, ne tirant son être que de la sainteté du contrat, ne peut jamais s'obliger, même envers autrui, à rien qui déroge à cet acte primitif, comme d'aliéner quelque portion de lui-même, ou de se soumettre à un autre souverain ${ }^{63}$. »

Le pacte induit une loyauté des individus qui fondent la communauté. Or, la pérennisation de la cité sera d'autant plus effective pour Rousseau que les membres vénèrent le pacte ${ }^{64}$. La profession de foi civile n'engendre donc pas simplement des «sentiments de sociabilité », elle contribue à sacraliser la cité et le pacte qui lui a donné naissance.

60. Burgelin P., La philosophie de l'existence de Rousseau, Paris, PUF, 1952, rééd. Genève, Slatkine, 1978, p. 162.

61. JACQUET Ch., La pensée religieuse de Jean-Jacques Rousseau..., op. cit., p. 163.

62. Rousseau J.-J., Du contrat social, Livre IV, Ch. VIII.

63. Rousseau J.-J., Du contrat social, Livre I, Ch. VII.

64. JACQUET Ch., op. cit., p. 176. 
La teneur de cette profession de foi jusqu'alors précisée par le Genevois constitue ainsi l'ensemble des « dogmes positifs». L'auteur en ajoute un dernier, qu'il qualifie de «négatif », à savoir l'intolérance. On sait que cette dérive avait été préalablement décrite par Rousseau dans sa critique du paganisme. L'intolérance est donc proscrite par la profession de foi civile. Celle-ci naîtrait d'un coupable exclusivisme religieux concernant la recherche du salut ${ }^{65}$. Le Vicaire abordait également « ce dogme contraire à la bonne morale» :

«C'est ce dogme horrible qui arme les hommes les uns contre les autres, et les rend tous ennemis du genre humain. La distinction entre la tolérance civile et la tolérance théologique est puérile et vaine. Ces deux tolérances sont inséparables, et l'on ne peut admettre l'une sans l'autre. Des anges mêmes ne vivraient pas en paix avec des hommes qu'ils regarderaient comme les ennemis de Dieu. »

L'ensemble des dogmes constituant la religion civile doit, de suite, nous interpeller sur la conciliation d'Émile et du Contrat social. La question s'impose d'autant plus naturellement, lorsque l'on sait que les articles de la religion civile - sauf un - demeurent semblables aux dogmes de la religion naturelle décrite par le Vicaire savoyard. Ces dogmes renvoient systématiquement à la recherche d'une profession de foi tempérée par la raison seule. Ainsi sont exposés les dogmes positifs (existence d'une divinité «puissante, intelligente, bienfaisante, prévoyante et pourvoyante, la vie à venir, le bonheur des justes, le châtiment des méchans ») ou négatifs (la simple intolérance). Pour autant, on sait également que le rôle bénéfique de la religion du Vicaire dans le champ politique a été aussitôt invalidé par Rousseau. Il faut s'arrêter ici un instant et définir les fonctions des deux religions décrites par le philosophe. Celles-ci divergent en tout point ${ }^{66}$, et ce, malgré la concomitance de l'écriture du chapitre ajouté au Contrat et d'Émile ${ }^{67}$. La religion de l'homme invoquée par le Vicaire traite de l'indispensable chemin de foi inhérent à toutes consciences, et tend à libérer ces dernières du joug de l'action coupable des hommes. Elle veut ôter le

65. «[...] par principe tout homme qui s'imagine qu'on ne peut être homme de bien sans croire tout ce qu'il croit, \& damne impitoyablement ceux qui ne pensent pas comme lui. » : Rousseau J.-J., Du contrat social.

66. Pour un nécessaire passage de la religion naturelle à la religion civile au sein de la cité politique, V. GouHier H., "La religion du vicaire savoyard dans la cité du Contrat social », in Études sur le Contrat social de Jean-Jacques Rousseau, actes des journées d'étude organisées à Dijon pour la commémoration du $200^{\mathrm{e}}$ anniversaire du Contrat social, Paris, Les Belles Lettres, 1964, p. 263-276.

67. V. Plongeron B., Théologie et politique au siècle des Lumières (1770-1815), Genève, Droz, 1973, p. 56. 
pouvoir temporel perverti et y substituer le dogme de la raison. La religion du Contrat - quant à elle - est avant tout celle du Législateur, elle avait été préalablement conceptualisée par Rousseau dans un chapitre portant sur celui-ci ${ }^{68}$. Cette religion - en ce sens - s'en trouve être indissociable de l'action étatique : elle doit être mise au service de l'instrument politique. L'universalité attachée à la religion du Vicaire demeure par essence limitée à cette recherche. Cette dualité emporte toute la singularité de la pensée de Rousseau. C'est que la profession de foi civile a un double intérêt : d'une part, elle offre une ouverture sur « la société générale du genre humain ${ }^{69}$ », d'autre part, elle se déploie dans une société, quant à elle, bien fermée, historiquement et géographiquement déterminée. Ainsi le Genevois mêle-t-il universalisme et particularisme ${ }^{70}$. Rousseau introduit ce particularisme à travers le dernier dogme positif de la profession de foi, celui de la sainteté du contrat social et des lois civiles. C'est que l'ordre de la raison n'est pas absolu $^{71}$, il peut parfois être tempéré par l'ordre juridique de la cité :

« Voit-on dans une rue ou sur un chemin quelque acte de violence et d'injustice. À l'instant un mouvement de colère et d'indignation s'élève au fond du cœur, et nous porte à prendre la défense de l'opprimé : mais un devoir plus puissant nous retient, et les lois nous ôtent le droit de protéger l'innocence.»

Nous savons désormais que la religion civile cultive le moralisme sans connaître les dérives belliqueuses et obscurantistes des vieux cultes païens. Elle proscrit la tentative théocratique et limite l'universalité à la citoyenneté. L'universalité anéantit toute haine de l'étranger, tandis que l'attache à la citoyenneté garantit un lien social prédominant en tempérant l'amour immodéré de son prochain et en y substituant la passion des lois, fut-elle simplement inscrite dans un inconscient. Les normes peuvent ainsi s'avérer malveillantes envers les cités politiques voisines, voire être aisément perçues comme injustes par les membres du pacte. Cela dit, le dernier

68. Pour mémoire, la religion civile figurait initialement en brouillon au dos des pages ayant trait au Législateur, Livre II, Ch. VII du Contrat social.

69. Intitulé du Ch. II du manuscrit de Genève.

70. DéChaux J.-H., art. cit., p. 277.

71. C'est que l'amour de l'ordre pourrait être mis en danger par le rôle joué par la conscience. Celle-ci pourrait trouver ces raisons absurdes, injustes, parfois tyranniques. Or on sait que les croyances générées par le Législateur répondent de la seule raison, c'est de cette dernière qu'elles tirent leur vérité existentielle. Il n'est ainsi guère étonnant que ces croyances, ces dogmes « essentiels à l'ordre moral » soient présentés par Rousseau comme «simples, en petits nombres, énoncés avec précision, sans explications ni commentaires » dans le Contrat. 
article tenant à la sainteté du contrat et des lois annihile toute tentative d'opposition. La vertu suscitée par le fait d'une religion naturelle demeure perpétuellement liée aux intérêts communs et reste conditionnée par le localisme: la loi est, in fine, souveraine. Ainsi, la religion civile procède d'une inclusion dans un ensemble «du céleste et du terrestre ${ }^{72} »$. Cette conciliation d'Émile et du Contrat social peut donc très bien être expliquée par cette nécessaire prise en considération du local et de l'universel. Pour Rousseau, l'imprégnation de la religion naturelle par les individus serait une étape préliminaire pour développer un véritable moralisme, sans les vices causés par les religions positives ${ }^{73}$. Toutefois, cette étape annoncerait, à terme, une projection dans le lien social ${ }^{74}$. À ce propos, le récit de la vie d'Émile est celui d'une éducation, d'un chemin de vie initiatique : il reste un préalable qui appelle d'autres horizons. L'universalisme entrouvert par les dogmes de la religion naturelle répond, une fois de plus, au plus parfait particularisme sur le plan politique. Une leçon est ici donnée à Émile : «Si je te parlais des devoirs du citoyen, tu me demanderais peut-être où est la patrie, et tu croirais m'avoir confondu. Tu te tromperais pourtant, cher Émile; car qui n'a pas une patrie a du moins un pays. Il $\mathrm{y}$ a toujours un gouvernement et des simulacres de lois sous lesquels il a vécu tranquille ${ }^{75}$.»

Ainsi le parfait universalisme n'est qu'affaire de perspective. L'homme sage, qui s'est laissé imprégner des dogmes de la religion naturelle, ne peut déployer sa vraie vertu que dans le lien social ; il "sait l'être [vertueux] malgré ses passions». De telle sorte, l'homme ne peut plus se révéler s'il s'affranchit du droit politique. Le choix que devra faire Émile en est une bonne parabole ${ }^{76}$. Rousseau lui annonce l'élévation à la citoyenneté : « [...] Mais cher Émile, qu'une vie si douce ne te dégoûte pas des devoirs pénibles, si jamais ils te sont imposés : souviens-toi que les Romains passaient de la charrue au consulat. Si le prince ou l'État t'appelle au service de la patrie, quitte tout pour aller remplir, dans le poste qu'on t'assigne, l'honorable fonction de citoyen. »

72. GuÉnARD F., précit, p. 32.

73. Waterlot G., Rousseau : religion et politique, op. cit., p. 93.

74. Déchaux J.-H., « Rousseau et la médiation symbolique entre les hommes : contribution à un individualisme structurel », art. cit, p. 284.

75. Rousseau J.-J., Émile ou De l'éducation, Livre V.

76. On retrouve également dans l'œuvre de Rousseau la profession de Julie, qui comprend que pour surmonter ses "intérêts particuliers", elle doit se tourner vers Dieu, lequel « donne un but à la justice et une base à la vertu». 
Ainsi, selon Rousseau, le vrai chrétien se réduit à un être chimérique. Il écrit dans sa préface de Narcisse que «s'il était permis de tirer des actions des hommes la preuve de leurs sentiments, il faudrait dire qu'il n'y a pas un seul Chrétien sur la Terre ${ }^{77} »$. Il écrira plus tard à M. de Malesherbes que « ce serait un grand hasard s'il y avait un seul Chrétien sur la Terre [...] le vrai Chrétien que la vie nous présente, même le meilleur, le plus proche de son idéal, est encore trop attaché à la Terre pour s'en désintéresser ${ }^{78}$ " selon le Genevois.

Ces éléments nous permettent d'appréhender de manière plus éclairée la profession de foi voulue par le philosophe. Nous savons qu'aux dogmes du Vicaire, Rousseau ajoute sa "sainteté du contrat social et des lois". Celle-ci, empruntée à la religion du simple citoyen, était présente dans les figures anciennes du paganisme. L'ajout de ce dogme unique, qui se joint à tous les autres, pourrait immédiatement renvoyer à une forme de synthèse réconciliatrice entre la religion de l'homme et celle du citoyen ${ }^{79}$. La tentative est verbalisée par Rousseau dans le manuscrit de Genève :

« Ainsi l'on réunira les avantages de la religion de l'homme et de celle du citoyen. L'État aura son culte et ne sera ennemi de celui d'aucune autre. Les lois divine et humaine se réunissant toujours sur le même objet, les plus pieux théistes seront aussi les plus zélés citoyens, et la défense des saintes lois sera la gloire du Dieu des hommes ${ }^{80}$.»

Cette forme de culte, dès lors, se manifeste par sa singulière position. Celle-ci semble perpétuellement attirée par deux pôles contradictoires. Il s'agit là d'un culte qui tente - c'est la grande ambition du philosophe - de concilier l'apparent irréconciliable. Rousseau nous a fait comprendre que les religions du citoyen, tirées de l'ancien paganisme ne peuvent être bonnes au sein de la cité, pas plus que le simple christianisme comme vecteur de lien social. Le philosophe avait bien compris que le Législateur ne pouvait raisonnablement envisager une société politique fondée sur l'un ou l'autre de ces pôles ${ }^{81}$. De la même manière, ceux-ci ne sauraient être associables, kata phusin. Il le mentionnera explicitement dans la Première lettre écrite de la Montagne: « Le patriotisme et l'humanité sont [...] deux vertus incompatibles

77. Rousseau J.-J., Narcisse ou L'amant de lui-même, préface.

78. Rousseau J.-J., Lettre à M. de Malesherbes, in Notes sur la Nouvelle Héloïse.

79. Déchaux J.-H., citant Simone Goyard-Fabre, parle ainsi d'« unité dialectique », art. cit., p. 274.

80. Rousseau J.-J., Du contrat social, ${ }^{\text {re }}$ version, Livre III.

81. Waterlot G., Rousseau : religion et politique, op. cit., p. 94. 
dans leur énergie, et surtout chez un peuple entier. Le législateur qui les voudra toutes deux n'obtiendra ni l'une ni l'autre : cet accord ne s'est jamais $\mathrm{vu}$; il ne se verra jamais, parce qu'il est contraire à la nature, et qu'on ne peut donner deux objets à la même passion ${ }^{82}$. »

Doit-on y voir une définitive aporie? C'est pourtant une nouvelle conceptualisation de la religion civile qui doit nous éclairer. Au terme de compromis $^{83}$, nous préférons rejoindre le constat de Ghislain Waterlot et traiter de "syncrèse chimique ${ }^{84}$ », d'une véritable mixtion créatrice. On sait que le Genevois était l'auteur d'Institutions chimiques ${ }^{85}$. Selon la définition de la syncrèse donnée par le philosophe, les religions de l'homme et du citoyen se sont altérées pour accéder à une nouvelle singularité conceptuelle : « La syncrèse consiste en de nouvelles mixtions de sorte que deux substances qu'on unit mêlées et confondues intimement en composent une troisième d'une forte union différente en nature de chacune de celles qui l'ont composée et où aucune d'elles n'est plus reconnaissable ${ }^{86}$. »

De la sorte, la présence de tous les dogmes de la religion du Vicaire - sauf un - change totalement la teneur de la profession de foi ${ }^{87}$, et suffit à engendrer une religion sui generis ${ }^{88}$. Celle-ci naît d'un tiraillement entre l'un et l'autre de ces antagonismes: humanité et patriotisme. Le cheminement de foi d'Émile illustre ce phénomène. Ainsi, la religion civile modifie en substance ces deux antagonismes tels qu'ils avaient été condamnés par Rousseau. De là, elle permet de les intégrer dans un corps altéré - ce qui n'est pas sans conséquence. La fonction de la religion civile paraît ainsi cristalliser une certaine tension. Cette dernière - plus qu'une spécificité - doit être considérée comme inscrite dans la substance du culte voulu par Rousseau. En ce point précis, la religion civile s'inscrit parfaitement dans la rhétorique propre au Genevois. Elle n'est qu'une manifestation pour l'homme de son élévation à la citoyenneté, qui doit structurer l'ordre social. Pour autant, l'état de nature reste un spectre omniprésent. En organisant la cité politique, la religion

82. Rousseau J.-J., Lettres écrites de la montagne, première lettre.

83. V. Derathé R., "La religion civile selon Rousseau », Annales de la société Jean-Jacques Rousseau, t. 35, 1959-1962, p. 170.

84. Waterlot G., Rousseau : religion et politique, op. cit., p. 89.

85. Pour Bruno Bernardi, « l'opposition de la mixtion et de l'agrégation a servi de paradigme à Rousseau pour la construction de ses concepts d'association et de corps politique » : in Bernardi B., "Pourquoi la chimie? Le cas Rousseau », Dix-huitième siècle, $\mathrm{n}^{\circ} 42$, 2010, p. 43.

86. Rousseau J.-J., Institutions chimiques, Partie IV, Ch. II.

87. TASSEL C., art. cit.

88. Culoma M., La religion civile de Rousseau à Robespierre, Paris, L'Harmattan, 2010, p. 33. 
civile appelle incidemment et paradoxalement au plus parfait universalisme, qu'elle révèle dans ses germes.

Cette construction intellectuelle appelle de telle sorte à une versatilité et à une adaptabilité, une «tension entre l'ouverture et la fermeture ${ }^{89}$ ». On l'a compris, la fermeture est rendue impossible avec l'introduction de l'ère du christianisme. L'ouverture complète est, quant à elle, tempérée par les particularismes, garantis par la « sainteté du contrat social et des lois ${ }^{90} »$. La religion civile, élément "syncrétique ", se situe donc entre ces deux pôles. De cette manière, la tension est déterminée par une contextualisation. Ainsi une conjoncture extérieure qui ne représenterait pas une menace pour le pacte porte vers une ouverture plus affirmée. Le contraire est également vrai : la mise en danger des particularismes exacerbe l'affirmation d'une citoyenneté sous le regard de la divinité. C'est tout le paradoxe et la subtilité de la pensée rousseauiste : ces particularismes demeurent les fruits d'une législation inhérente à l'action de la providence, qui doit elle-même être reconnue par l'humanité tout entière. Le contrat est donc, en ce sens, doublement sacralisé. Il s'inscrit sous l'égide d'une foi universelle, mais entraîne également la passion de la législation qui l'engendre. Le parfait universalisme introduit, au fond, la projection du citoyen dans la cité politique : «Sitôt que la puissance législative parle, tout rentre dans l'égalité ; toute autorité se tait devant elle; sa voix est la voix de Dieu sur Terre ${ }^{91}$. »

En guise de conclusion, nous pouvons citer ces quelques mots du philosophe, tirés de ses Principes du droit de la guerre. Ceux-ci viennent rappeler ce conflit permanent, propre à la nature de l'homme :

« La première chose que je remarque, en considérant la position du genre humain, c'est une contradiction manifeste dans sa constitution, qui la rend toujours vacillante. D'homme à homme nous vivons dans l'état civil et soumis aux lois; de peuple à peuple chacun jouit de la liberté naturelle; ce qui rend au fond notre situation pire que si ces distinctions étaient inconnues. Car, vivant à la fois dans l'ordre social

89. Waterlot G., La religion civile de Jean-Jacques Rousseau..., précit., Deuxième partie.

90. On sait que Rousseau se méfiait d'une universalisation des choses, et de son aspect désocialisant. Ainsi peut-on lire dans le manuscrit de Genève : "Par où l'on voit ce qu'il faut penser de ces prétendus cosmopolites, qui justifiant leur amour pour la patrie par leur amour pour le genre humain, se vantent d'aimer tout le monde pour n'avoir droit d'aimer personne. » : RousSEAU J.-J., Du contrat social, première version.

91. Rousseau J.-J., Considérations sur le gouvernement de Pologne, Ch. VI ; V. Waterlot G., La religion civile de Jean-Jacques Rousseau..., précit., Deuxième partie. 
et dans l'état de nature, nous sommes assujettis aux inconvénients de l'un et de l'autre, sans trouver la sûreté dans aucun des deux. »

La religion civile, en tentant d'intégrer le patriotisme et l'humanité, révèle ce déchirement, qui fonde tout le discours rousseauiste. Le culte voulu par le philosophe réussit-il à surmonter cette apparente insolubilité ? En traitant de solution compromissoire, la réponse serait très certainement négative; en envisageant la solution syncrétique, nous pouvons croire en la résolution d'un conflit. 